\title{
Une revue à l'affût du monde? L'Éducateur comme relais des transferts et métissages pédagogiques en Suisse romande (1865-1890)
}

\section{Alexandre Fontaine}

Cet article 1 a pour dessein d'analyser les modalités de transferts de savoirs qui s'opèrent durant le second dix-neuvième siècle en Suisse romande. En plaçant L'Éducateurla revue des instituteurs romands fondée en 1865 - au centre du questionnement, il s'agit de déconstruire les phénomènes d'emprunts tant régionaux que transnationaux et de montrer par là même les résistances et les enjeux centraux de la circulation des savoirs via le puissant réseau des revues pédagogiques.

Dès le milieu du $18^{\mathrm{e}}$ siècle, la presse d'éducation permet la circulation de savoirs pédagogiques dans une Europe scolaire en construction. Les méthodes se discutent, s'échangent et se resémantisent (Espagne, 1999) en fonction des besoins des contextes particuliers. On rappellera avec Alain Choppin (2008) que la revue a longtemps occupé les fonctions de livre du maître ou du professeur et tenu de ce fait un rôle déterminant. De plus, comme le souligne Pierre Caspard (1981), "la presse constitue un maillon indispensable dans la connaissance de ce qu'a été, pendant près de deux siècles, le système d'enseignement, car elle présente à la fois l'espace où se déploient et le point où se focalisent tout un ensemble de théories et de pratiques éducatives d'origine tant officielle que privée» (p. 8).

L'échange massif de savoirs scolaires sur le continent laisse à penser que la plupart des revues pédagogiques se sont muées en interprètes d'une pédagogie toujours davantage métissée. Ce constat s'avère particulièrement probant pour L'Éducateur - l'organe de la Société des instituteurs de la Suisse romande (dorénavant SIR) - qui peut être considéré comme un lieu de mémoire interculturelle pour diverses raisons. Rappelons en premier lieu avec Pierre Nora (1984) que ces lieux de mémoire, "du plus matériel et concret, comme les monuments aux morts et les Archives nationales, au plus abstrait et intellectuellement construit, comme la notion de lignage, de génération, ou même de région et d' "homme-mémoire» [...] commandent l'économie du passé dans le présent» (p. VII-VIII, cité par Bugnard, 1998/1999, p. 131). C'est précisément cette "économie du passé dans le présent» qui conditionne l'étude de $L ' E ́ d u$ - 
cateur. Ainsi, il s'agit tout d'abord de souligner l'exceptionnelle ouverture internationale que le premier rédacteur Alexandre Daguet (1816-1894) a insufflé à son contenu. Le périodique regorge en effet de références étrangères et dans cette perspective, interroger la masse importante d'articles et de recensions consignées sur la France fait sens. Bien entendu, la réciproque se vérifie clairement. La gigantesque bibliothèque de Jean-Jacques Rapet (1805-1882), qui est à l'origine de la constitution du Musée pédagogique, incarne également un lieu de mémoire français de la pédagogie suisse. Acheté par l'État français (loi du 5 juin 1880), le fonds de cet éminent spécialiste de la pédagogie helvétique constitue une collection unique au monde, surtout en ce qui concerne les ouvrages relatifs à la pédagogie suisse dès lors instituée comme référence en France par Ferdinand Buisson ${ }^{2}$. Que l'on songe également aux dizaines d'articles reproduits dans le Manuel général de l'instruction primaire par Charles Defodon (1832-1891) pour rendre compte des congrès romands auxquels il participait activement. De ce fait, le chercheur intéressé par les conférences pédagogiques suisses trouvera davantage d'informations sur la question dans la presse d'éducation française que dans les revues romandes. Cet usage transnational des revues mérite donc d'être souligné3.

Dans la présente contribution, nous examinerons certains aspects de la construction transnationale des théories et pratiques éducatives qui circulent en Occident pour mesurer leur acculturation dans l'espace romand. En plaçant L'Éducateur au centre de la discussion, nous examinerons dans un premier temps les principales caractéristiques éditoriales de l'organe des instituteurs romands sous la rédaction de Daguet, avant de nous intéresser à l'une de ses collaboratrices. Caroline Progler, oubliée aujourd'hui, fut une médiatrice particulièrement active qui a utilisé ce périodique pour transférer en Romandie le système du Kindergarten développé par Friedrich Fröbel (1782-1852). Puis, en questionnant les relations pédagogiques alémano-romandes, nous analyserons l'impact de la construction identitaire sur la représentativité des emprunts transrégionaux. Enfin, nous mettrons en perspective les principales références étrangères qui transitent dans L'Éducateur entre 1865 et 1890.

\section{Mission et caractéristiques éditoriales de L'Éducateur}

Lors de la publication du numéro initial en janvier 1865, le comité directeur ${ }^{4}$ de L'Éducateur ne cache pas son ambition de supplanter les revues cantonales existantes afin de constituer un monopole: «Cette union est nécessaire encore si l'on désire (et on doit le désirer) que la feuille de la Suisse romande l'emporte sur les feuilles purement cantonales qui l'ont précédée dans la carrière» $(1 / 1865$, p. 3). Il faut dire que dès les années 1850 , des voix se font entendre pour lutter contre tout esprit cantonaliste. Qui mieux qu'Alexandre Daguet pouvait 
mener cette œuvre de médiation? Intellectuel doué d'un savoir encyclopédique, historien national, Daguet théorise la pédagogie libérale-nationale romande du second dix-neuvième siècle. En intériorisant les idées de tolérances religieuses, d'éclectisme et d'universalité de la pédagogie développées par son maître le père Grégoire Girard (1765-1850)5, il œuvre pour le rassemblement corporatif des instituteurs de Suisse française. Porté par un catholicisme irénique ou de conciliation, Daguet s'incarne ainsi en porte-drapeau de la phalange libérale-nationale de la pédagogie romande, alors que Raphaël Horner symbolise sa fraction catholique au travers du Bulletin pédagogique publié dès janvier 1872. Jean-Marie Barras (2005) rappelle à ce sujet que «si les idées pédagogiques que présentera le Bulletin seront le plus souvent novatrices, voire d'avant-garde, l'idéologie en sera carrément conservatrice» (p. 40).

L'Éducateur - toujours en activité aujourd'hui sous la forme d'un mensuel - paraît du temps de Daguet le $1^{\text {er }}$ et le 15 de chaque mois et se divise en deux parties principales: une première consacrée aux articles de fond, théoriques et une seconde dévolue à la correspondance, aux notices bibliographiques et aux comptes-rendus. Une partie pratique fait son apparition en 1869, avec des exercices de mathématiques ou des dictées à utiliser directement en classe.

Ce bimensuel est par ailleurs pensé par son rédacteur comme un banc d'essai des divers systèmes étrangers, comme un lieu d'ouverture qui sert avant tout à élargir l'horizon des instituteurs romands. Daguet promeut l'éducation d'un corps qu'il veut armer en termes de méthodes pédagogiques. Il entreprend la diffusion d'une theoria éclectique, seule susceptible selon lui d'instituer un "code commun» dans chaque partie de la Suisse romande et arracher ainsi l'instituteur à son isolement:

La lecture d'une feuille pédagogique bien dirigée fait à l'esprit de l'instituteur l'effet d'une eau pure et savoureuse à celui qui a soif. L'intelligence est avivée, le savoir accru, la méthode perfectionnée, le cœur agrandi et ennobli. Quel est l'instituteur qui ne serait satisfait de voir ce que les autres font, ce qui se passe autour de lui et au-delà, de quelles questions on s'occupe et quels progrès s'accomplissent. Il y a cependant des instituteurs qui ne lisent aucun journal scolaire et ne s'inquiètent pas le moins du monde de ce qui se fait en pédagogie. Ils tiennent leur école demain comme hier et ne pensent même pas qu'ils aient à apprendre quelque chose: ils taxent de théories creuses ou arbitraires tout ce qui est en dehors de leur horizon ou contrarie leur routine, oubliant le fameux mot de Goethe, le coryphée de la littérature allemande: "Qui n'avance pas recule». Nous ajouterons: celui qui n'a pas l'habitude de porter ses regards au-delà de la banlieue où il se trouve, devient myope et sans cœur (Daguet, 1880a, p. 50).

Entre 1865 et 1890, L'Éducateur possède un nombre d'abonnés relativement stable, ce qui équivaut à une moyenne de 1200 tirages. Il touche ainsi la moitié du corps enseignant romand et est envoyé à plusieurs personnalités de l'Europe 
pédagogique (F. Buisson, G. Compayré, G. Sacchi, J.-J. Campion, V. di Castro, V. Stoy, etc.). Il emprunte sa devise Dieu-Humanité-Patrie à l'École cantonale de Fribourg dirigée par Daguet entre 1848 et 1857, devise qui imprime le cadre idéologique de sa ligne rédactionnelle. Signalons encore que L'Éducateur a la particularité d'être une revue itinérante, changeant de lieu et de maison d'édition tous les deux ans et parcourant ainsi les principales villes de Suisse romande.

Lors de la constitution de la première équipe rédactionnelle, Daguet cherche avant tout à s'entourer de personnalités «fiables». Il s'agit de prendre toutes les précautions afin de ne pas répéter l'expérience douloureuse de l'École cantonale de Fribourg, où les luttes politiques entre les membres du corps enseignant ont jeté le doute sur ses capacités à diriger une équipe pédagogique. Fort des apprentissages retenus lors de ce qui s'apparente à une fronde, le noyau initial des collaborateurs de la revue regroupe, pour l'essentiel, des anciens étudiants de Daguet proches des milieux libéraux-nationaux catholiques et protestants, parmi lesquels on trouve Auguste Biolley, Alexis Bourqui, Joseph Rey, Pierre Ducotterd et Jules Paroz. Ainsi, contrairement à un Ferdinand Buisson qui a dû jongler avec des monarchistes dans la première équipe rédactionnelle de son Dictionnaire de pédagogie et d'instruction primaire (Dubois, 1995 \& 2000), Daguet a pu, globalement, s'appuyer sur l'homogénéité idéologique de ses collaborateurs, au nombre de trois cents sous sa période rédactionnelle. Dans cet environnement essentiellement masculin, une poignée d'éducatrices vont jouer un rôle déterminant dans la circulation de savoirs pédagogiques spécifiques et méritent de ce fait un éclaircissement.

\section{Caroline Progler et la diffusion de "l'évangile fröbelien»}

On s'en doute, énumérer les collaboratrices de L'Éducateur sous la période rédactionnelle d'Alexandre Daguet relève de l'anecdotique. Outre la notice sur les grandes difficultés de l'éducation morale d'Hortense Ray, décédée tragiquement en 1865 ou quelques rares poèmes de la neuchâteloise Amélie Pernod, les femmes et leurs revendications demeurent clairement au second plan de la phase initiale de la revue (1865-1889). Plusieurs arguments sont avancés par les instituteurs eux-mêmes: l'un relève d'un manque d'aptitude, l'autre évoque la faiblesse féminine. Il faut dire que l'angoisse pernicieuse d'un déclassement en faveur des institutrices traîne ici et là, amères revendications que l'on retrouve sporadiquement dans le courrier des lecteurs.

L'une d'entre elles va néanmoins réussir à émerger de ce groupe, pour faire connaître et propager un système pédagogique germanique en Suisse romande. Caroline Progler (?-1886) représente la plume genevoise qui intervient le plus souvent dans $L^{\prime} E$ ducateur, avec trente-sept contributions entre 1868 et 1884. Entièrement dévouée à la diffusion de l'œuvre de Fröbel, elle a donc trouvé dans 
ce périodique une plateforme particulièrement appropriée pour diffuser la doxa fröbelienne en Suisse occidentale.

\section{La Romandie comme carrefour de la diffusion trans- nationale de l'œuvre de Friedrich Fröbel}

L'histoire de l'éducation préscolaire et la diffusion de la pédagogie de Friedrich Fröbel en Suisse romande ont été remarquablement traitées par Michèle Schärer (2008). On pourrait encore s'interroger sur le régime circulatoire de la méthode fröbelienne. À cet égard, il faut souligner le rôle de self-service que la Suisse a joué dans la propagation des principes du pédagogue thuringien. Édouard Raoux, professeur à l'Académie de Lausanne, correspondant de Victor Cousin, symbolise sans nul doute un médiateur zélé de l'œuvre de Fröbel en Suisse romande. En 1861, il lance une revue mensuelle intitulée L'Éducation nouvelle ou méthode Froebel dans le but de vulgariser et répandre la méthode. Il s'entoure de collaborateurs européens prestigieux, parmi lesquels on trouve la baronne von Marenholtz-Bülow, le $\mathrm{D}^{\mathrm{r}}$ Karl Schmidt et le $\mathrm{D}^{\mathrm{r}}$ Diesterweg de Berlin${ }^{6}$.

On ne saurait trop souligner que la diffusion européenne de Fröbel fut réalisée en grande partie par des femmes et que Genève y détient une place de choix: «Découragé, voyant que les pédagogues de profession l'accueillaient avec défiance, que les hommes de science refusaient de le prendre au sérieux, que les pouvoirs publics restaient indifférents, Fröbel résolut de s'adresser dorénavant aux femmes, et de remettre sa cause entre leurs mains», souligne James Guillaume (1911). Ces ambassadrices se sentirent investies d'une responsabilité incontestable. Ce faisant, elles endossèrent les fonctions de missionnaire afin de faire tomber les préjugés qui courraient à l'égard de l'œuvre de leur mentor. Ne pouvait-on pas lire dans le dictionnaire pédagogique de Max Ricci: «Jardin d'enfants: serre chaude pour le développement prématuré de l'enfance» (Progler, 1882, p. 66).

C'est Henri-Frédéric Amiel qui, en novembre 1865, alerte son ami Daguet sur les qualités de l'œuvre de Fröbel, qu'il s'agit de présenter rapidement aux enseignants romands: "Pourquoi ne parle-t-on pas de Fröbel dans L'Éducateur? J'ai toujours cru et dit que les trois ou quatre premières années de la vie sont les plus importantes, et c'est la vue capitale de ce pédagogue, un des plus pénétrants qu'il y ait eu» (Amiel, 1865). Malgré cette invitation, Daguet reste d'abord sceptique à l'idée d'ouvrir ses colonnes aux procédés du "Pestalozzi allemand», notamment en raison du caractère particulièrement onéreux de cette méthode pédagogique $(1867$, p. 263). De plus, il contribua à colporter un certains nombres d'idées préconçues comme l'aspect ludique des Kindergarten, composante combattue par ses adeptes puisque l'on préférait parler de «travail déguisé». Néanmoins, Caroline Progler, son initiatrice Adele von Portugall et Daguet lui-même démocratisèrent la méthode fröbelienne par une série d'articles diffusés dans L'Éducateur: 
1867 - Les jardins d'enfants, p. 282-285.

1869 - [Frœbel à] l'exposition scolaire de Lausanne, p. 363-365.

1871 - La méthode Frœbel et ses adhérents, p. 198-201.

1877 - Le rapport de Mlle Progler sur la méthode Fröbel, p. 316-317.

1881 - Le système Frobel au Congrès international de l'Enseignement de Bruxelles, p. 50-52.

1881 - Les jardins d'enfants sans jardins, p. 305-309.

1882 - Les jardins d'enfants, p. 53-54

1882 - Le centenaire de Frobel, p. 65-68.

1882 - Les jardins d'enfants de Berlin, p. 102.

1884 - La cause de Frobel, p. 265.

On notera par ailleurs que la dynamique interculturelle et l'extrême mobilité de ces femmes ont largement favorisé le prêche de ce que l'on appelait, selon le mot de Michelet, «l'évangile fröbelien». Si l'on s'en tient aux données biographiques, rappelons par exemple que la baronne Adele von Portugall (1828-1910) étudia la méthode Fröbel à Dresde, se rendit à Liverpool avant de s'installer à Genève en 1864. Après dix ans passés à diriger les écoles de Chantepoulet, elle partit pour Mulhouse vers la fin 1873, revint sur les bords de l'Arve en 1876 pour prendre la direction des écoles enfantines de Genève avant de s'exiler à Naples en 1884. Un article qui a paru dans le Journal de Genève du 9 août 1911, signé D., fait remarquer que "pour comprendre les pérégrinations de $\mathrm{M}^{\text {me }}$ de Portugall, il ne faut pas oublier qu'elle est un apôtre; elle en a l'énergie, la conviction et l'autorité, elle en a le tempérament migrateur et remuant» (p. 4).

Bien qu'il subsiste encore de nombreuses zones d'ombre dans l'itinéraire de Caroline Progler (notamment sur ses origines), les données connues laissent entrevoir les grandes lignes de son œuvre de médiation. Elle rencontre Adele von Portugall à Genève en 1867. En 1875, lorsqu'elle s'engage à rédiger le rapport sur les écoles enfantines du futur congrès de Fribourg, elle dirige un jardin d'enfants à Mulhouse. Est-ce $\mathrm{M}^{\text {me }}$ de Portugall qui l'entraîne avec elle à Genève, écourtant son exil alsacien pour prendre la direction des écoles enfantines des bords de l'Arve? À l'automne 1876, Progler ouvre des cours de français, d'arithmétique, de géographie, d'histoire, de sciences naturelles, d'allemand, d'anglais et d'ouvrages à l'aiguille (méthode Kettiger-Schaldenfeld) qu'elle donne chez elle, 8 place du Molard. Nous l'avons vu, en 1884, les deux éducatrices s'installent à Naples afin d'y établir des jardins d'enfants. Elles apportent ainsi leur coopération à l'œuvre de $M^{\text {me }}$ Schwab, fondatrice d'une vaste institution de jeunes filles placée sous l'égide de la reine d'Italie. Caroline Progler y meurt le 19 janvier 1886, des suites d'une longue maladie.

Si la diffusion opérée par Progler dans L'Éducateur se révèle tout à fait visible, certains emprunts demandent une lecture plus attentive, du fait surtout de la construction identitaire qui tend à dévaloriser certains transferts afin de privilégier les aspects du cru. Avant d'analyser la circulation globale de savoirs pédagogiques étrangers dans L'Éducateur, nous étudierons la dynamique contrastée des relations avec les collègues de Suisse allemande. 


\section{Crispations interrégionales et circulations dissimulées}

Il n'est pas inutile de rappeler qu'un groupe d'instituteurs, composé de Vaudois, de Neuchâtelois, de Jurassiens, de Genevois et de Fribourgeois assiste à la $5^{\mathrm{e}}$ réunion du Lehrerverein (Société pédagogique de Suisse allemande) à Berne les 9 et 10 octobre 1863. Daguet (1866) évoque à ce titre que les régents romands, «se sentant un peu perdus au milieu de sept cents collègues germains, éprouvèrent le besoin de faire plus ample connaissance, d'échanger leurs idées, de se communiquer leurs sujets de crainte et d'espérance, puis de donner au lien sympathique qui s'établissait entre eux, une certaine fixité » (p. 7). Un comité d'initiative, présidé par l'instituteur Frédéric Villommet, se constitue autour d'un groupe de Neuchâtelois ${ }^{7}$, dont le principal défi est de regrouper les cantons romands dans un faisceau pédagogique commun et ceci malgré de profondes différences structurelles. Si Neuchâtel et Vaud se sont déjà constitués en sociétés pédagogiques cantonales, Genève se fédère en 1867 . Fribourg fonde sa société en novembre 1871, crée son propre organe, le Bulletin pédagogique en janvier 1872 et décide de quitter un giron romand jugé par trop libéral et antichrétien en $1877^{8}$. Quant au Valais, toutes les démarches restèrent infructueuses jusqu'à la fondation, un siècle plus tard, de la SPVal le 9 juin 1968.

Au moment de créer un espace pédagogique romand, on envisagea en premier lieu une fusion des enseignants romands avec ceux du Lehrerverein, mais la langue allemande demeurait un fossé infranchissable:

La Suisse allemande peut déjà nous présenter les bienfaits d'une association pédagogique. Certes, nous n'eussions pas mieux demandé, pour la facilité de notre tâche, que de nous joindre à elle et de profiter ainsi de ce qui existe. Mais, après avoir mûrement étudié la chose, il nous est apparu qu'il serait de toute impossibilité de réunir dans une même assemblée les instituteurs des diverses langues de la Suisse. En effet, il ne peut être question de faire entrer dans une société de langue allemande des hommes qui n'en comprennent pas le premier mot, qui ne pourront ainsi prendre part en connaissance de cause aux délibérations, et dont le nombre d'ailleurs sera toujours dominé par la majorité qui les environnera. Nous pourrons d'ailleurs nous allier en tout temps avec nos frères allemands; ce que nous réclamons ici, c'est le maintien de notre individualité. D'autre part, ce qui importe surtout d'avoir avec eux, c'est une communauté d'intentions. Or, notre programme est le même (Savary, 1914, p. 7).

Après une courte période de gestation, deux cent cinquante instituteurs romands officialisent la fondation de la SIR à Neuchâtel. Ce 26 septembre 1864 marque le point de départ d'une mémoire collective des instituteurs de la Suisse occidentale. Presque trente ans après la fondation de la Société d'histoire de la Suisse romande en 1837 - Alexandre Daguet en est membre-fondateur - on 
assiste donc à une seconde marche vers l'autonomie régionale, pédagogique cette fois-ci. On ne saurait trop relever l'importance considérable de ce rattachement des sociétés pédagogiques cantonales existantes en un faisceau unique, quand on sait combien la prééminence des cantons en matière d'instruction publique est prégnante sur le territoire helvétique.

\section{Des relations confédérales en decrescendo?}

Comment évoluèrent, à moyen terme, les relations avec les "frères» d'outreSarine? Sur la base d'une étude sur les relations confédérales dont on peut retracer le fil à partir de L'Éducateur et des comptes-rendus de congrès, on peut affirmer qu'il existe, dans les années consécutives à la fondation de la SIR, une réelle volonté d'échange et de rapprochement. En 1865, le Landammann Vigier, de Soleure, adresse une invitation à assister au congrès du Lehrerverein dans sa ville. Daguet (1865) s'empresse de répondre que «tout en ayant à cœur de s'affirmer et de faire acte d'autonomie, dans le domaine pédagogique et intellectuel en général, la Suisse romande tient aussi à honorer et regarde comme un devoir de rester fermement unie à la Suisse allemande, sa sœur aînée en liberté et en patriotisme» (p. 59). Une vingtaine d'instituteurs romands y prend part, comme à celui de St-Gall en 1867.

Pourtant, à y regarder de plus près, les coopérations et les échanges, notamment lors des congrès respectifs, s'avèrent peu conséquents, malgré d'innombrables appels à l'union confédérale. Dans L'Éducateur, on trouve déjà quelques mouvements d'humeur dès 1869. En 1871, Daguet (1871b) déplore publiquement la piètre qualité des relations interrégionales: «L'isolement, un isolement déplorable continue à peser d'un point de vue intellectuel surtout entre les Suisses de langues différentes. Cet isolement est la plaie de la Suisse. La politique seule semble avoir le privilège de mettre en contact la Suisse allemande et la Suisse romande» (p. 87).

Médiateur, comme Eugène Rambert, et trait d'union entre les deux berges de la Sarine, Daguet tente, lors du congrès de la SIR de 1879 à Lausanne, d'insuffler une nouvelle dynamique aux relations pédagogiques confédérales, qui se résument à une courtoise indifférence réciproque. Malgré tout, les rapports avec la Suisse orientale deviennent toujours moins conséquents, noyés dans la masse des échanges internationaux. À l'heure du bilan, Daguet exprime en 1886 que:

Depuis la fondation de L'Éducateur, nous avons constamment eu en vue de servir de trait d'union entre les confédérés des trois langues et travaillé au rapprochement, à l'échange des idées, sans toujours y parvenir. Nos frères allemands eux-mêmes, qui nous accusent parfois de tiédeur et se plaignent qu'on n'assiste pas en nombre à leur Lehrertag, viennent-ils beaucoup aux nôtres? Je traduis ou j'extrais souvent leurs articles; traduisent-ils ou extraient-ils les nôtres? (Compte-rendu de Porrentruy, 1886, p. 74). 
Voici pour le discours dominant. Pourtant, dans les faits, la place accordée à la Suisse allemande pédagogique dans L'Éducateur ne semble pas si maigre et effacée qu'elle en paraît dans le discours. Entre 1865 et 1889, on dénote quelque cent trente entrées (articles de fond ou chroniques scolaires) spécifiquement consacrées à la Suisse alémanique. Certes, on recense un nombre important d'articles consacrés aux divers synodes du canton de Berne, rapportés par le Jurassien Henri Gobat. Toutefois, on relate et on analyse systématiquement les temps forts des discussions menées lors des Lehrertage. De plus, Daguet publie un certain nombre de biographies de pédagogues alémaniques. On remarque d'abord une notice sur le Thurgovien Johannes Jakob Wehrli (1790-1855), une autre sur l'éducateur démocrate appenzellois Heinrich Grunholzer (18191873), le directeur de l'école normale de Münchenbuchsee. Daguet sauve également Josef Anton Federer (1794-1868), Thomas Scherr (1801-1870) et Johann Rudolf Steinmüller (1772-1835) d'un oubli certain en faisant partager leurs trajectoires aux régents romands. C'est dans ce même souci de «sauvetage national» que le rédacteur de L'Éducateur soumet, en 1885 un Coup d'ail sur les Pédagogues et Écrivains pédagogiques de la Suisse allemande.

Néanmoins et malgré cette présence alémanique distincte dans L'Éducateur, on peut affirmer qu'à trop vouloir exiger son autonomie, il est probable que la SIR et sa revue se sont peu à peu distancées des projets initiaux d'union, se satisfaisant d'inviter les confrères d'outre-Sarine à participer à leurs congrès, ou à réhabiliter de temps à autre quelques figures de la pédagogie alémanique. Car si Lausanne est au $18^{\mathrm{e}}$ siècle la plus cosmopolite des villes suisses pour appartenir à l'Europe plutôt qu'à Berne, peut-être bien que L'Éducateur s'ouvre à l'international pour se départir d'une «majorité germanique [qui] ferait sentir à la minorité welsche le poids de sa force numérique et d'une culture supérieure à certains égards» (Daguet, 1869, p. 219).

Comme l'a montré Hofstetter (2010), Genève et la Suisse ont occupé une place privilégiée, dès le $19^{\mathrm{e}}$ siècle, dans le mouvement des idées pédagogiques ainsi que dans le processus constitutif des sciences de l'éducation. Pensée comme un lien entre les peuples du Nord et du Midi, la Suisse romande pédagogique s'est-elle dès lors édifiée dans une logique d'appropriation de ces références étrangères?

\section{Quelques références étrangères absorbées par L'Éducateur}

Unique rédacteur des chroniques scolaires, Alexandre Daguet décide, au gré de ses lectures de revues étrangères qu'on se presse de lui envoyer ou d'échanger contre $L^{\prime} E$ ducateur, des articles à traduire ou des informations à diffuser. Afin d'inventorier les références étrangères présentes dans la revue romande, nous avons mené une recherche thématique sur vingt-cinq ans (1865-1890) en 
sondant les articles de fond et les chroniques. Nous avons ensuite réparti ces contributions $^{9}$ dans quatre catégories:

1. Méthodes et cours théoriques

2. État de l'instruction publique dans le monde

3. Revue de la presse pédagogique étrangère

4. Expositions universelles/nationales et congrès pédagogiques

Méthodes et cours théoriques diffusés dans L'Éducateur

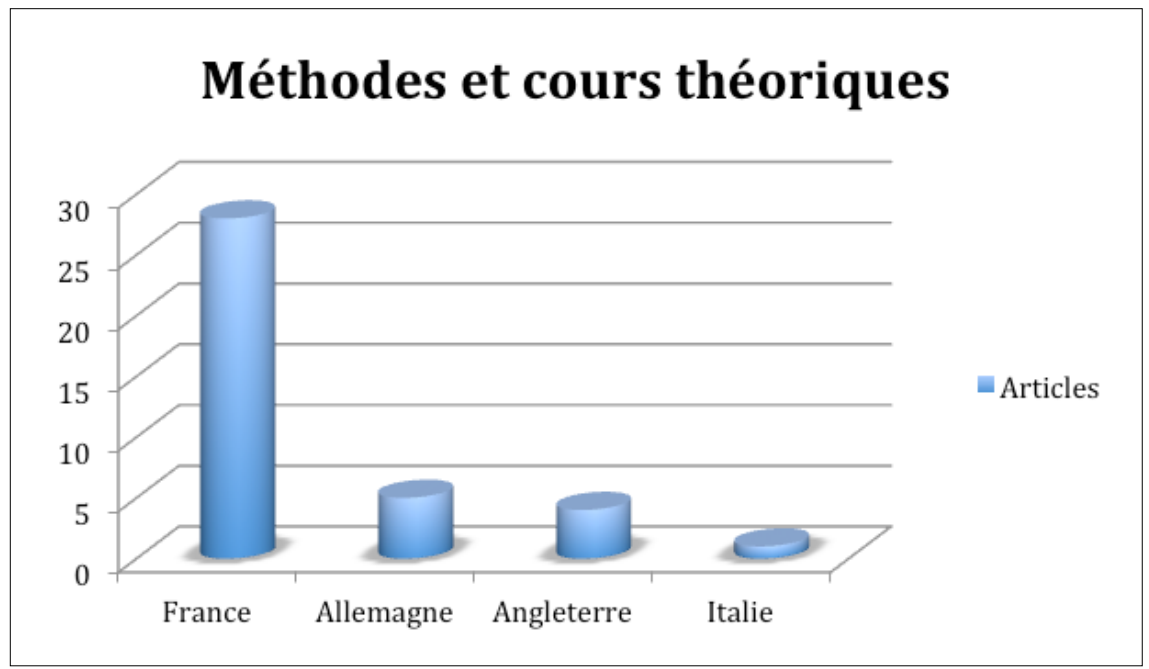

Tableau 1: Méthodes et cours théoriques recensés dans L'Éducateur

Dans cette première catégorie, nous avons regroupé les méthodes étrangères diffusées dans L'Éducateur, comme la catéchisation de Dinter (1868) par exemple, ainsi que les cours théoriques présentés par Daguet comme des «modèles» - ou des "contre-modèles» d'ailleurs - à adopter ou à rejeter.

On recense presque une trentaine d'articles en provenance de l'Hexagone. À dire vrai, la pédagogie française s'impose dans $L$ 'Éducateur dès les premiers écrits de Gabriel Compayré (Histoire critique de l'Éducation en France, recensée en 1880). Elle monopolise dès lors la revue romande jusqu'à l'éviction de Daguet en 1889 (quatre articles sur la pédagogie à l'usage de l'enseignement primaire de Paul Rousselot en 1882, un article sur l'Histoire de la pédagogie de Compayré en 1883, quatre sur son Cours de 1886, un article sur la méthode active de Henri Marion en 1888). Si la pédagogie républicaine est globalement encensée par Daguet, la science de l'éducation de Herbert Spencer constitue au contraire une sorte "d'anti-modèle». Daguet attaque avant tout l'utilitarisme et le positivisme de la théorie éducative de Spencer et d'Alexander Bain dans une série de trois 
articles parus en 1880, et légitime ses vues en publiant une critique similaire émise par Müller de Berlin (1885). Il incite néanmoins les instituteurs romands à parcourir ces ouvrages, en concédant que certains passages s'avèrent remarquables.

États de l'instruction publique dans le monde

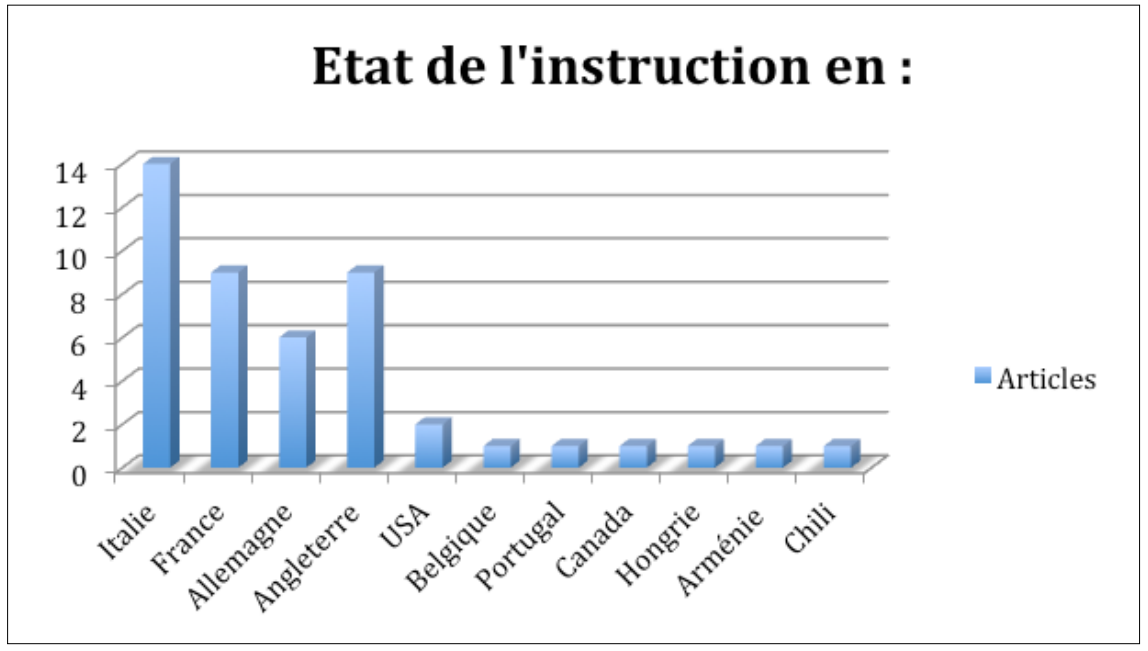

Tableau 2 : État de l'instruction publique dans le monde

Dans ce second groupe sont répertoriés les multiples «coups d'œil» et «états de l'instruction» dans les diverses parties du monde. Assez curieusement, ce sont les progrès de la pédagogie italienne qui demeurent le plus souvent étudiés et commentés dans L'Éducateur. Par ailleurs, on mesure très clairement quelles nations sont surveillées par Daguet. Il serait impensable de ne pas discerner une innovation opérée dans l'une de ces quatre grandes nations européennes. De ce fait est-il déterminant de dresser, de temps à autre, un panorama de leurs progrès pédagogiques afin de renouveler les connaissances romandes.

Les articles consacrés au système scolaire étasunien présentent également de l'intérêt. En effet, celui-ci se verra fortement dévalorisé dans les colonnes de L'Éducateur jusqu'au milieu des années 1870 :

Mais en dépit de tous ces avantages, l'école populaire anglo-américaine a, pédagogiquement parlant, peu de chose à proposer à l'imitation des écoles européennes. Le mécanisme règne dans presque toutes les classes, et l'enseignement repose tout entier sur la récitation et sur les livres (text-books). La méthode et la discipline y sont nulles. L'habitude d'employer des institutrices au lieu d'instituteurs semble avoir pris naissance dans une économie mal entendue, plutôt que par suite d'une préférence fondée sur des principes. Ces 
institutrices improvisées se marient au bout de quelques années, et font place à d'autres débutantes qui disparaissent à leur tour (Daguet, 1871a, p. 69).

La représentation du système américain se modifie et s'inverse même, grâce au regard neuf posé par Ferdinand Buisson suite à son Rapport (1878) sur l'exposition universelle de Philadelphie en 1876. Au demeurant, ce voyage de Buisson coïncide avec une nouvelle stratégie internationale de la France, qui déplace peu à peu son «œil pédagogique» de la Suisse (entre autres) vers les États-Unis.

Revue de la presse pédagogique étrangère

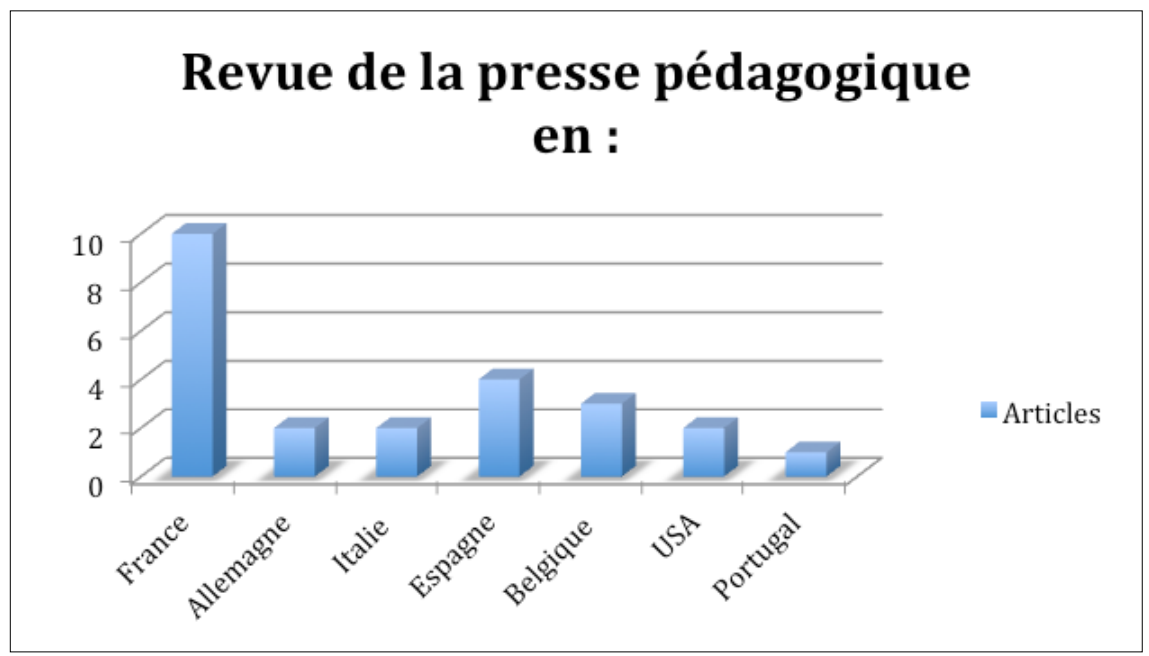

Tableau 3 : Revue de la presse pédagogique étrangère

Dans cette troisième catégorie, nous avons recensé les revues de la presse scolaire étrangère. Il s'agit plus particulièrement de commentaires spécifiques consignés suite à la lecture des revues échangées contre L'Éducateur. À nouveau, la place de la France s'avère nodale, notamment grâce à la coopération que Daguet entretient avec le Manuel général de l'instruction primaire de Charles Defodon et la Revue pédagogique dès sa parution en 1878 . On notera les bonnes relations entretenues avec l'Espagne, pays duquel Daguet reçoit régulièrement les meilleures feuilles éditées par des républicains comme Emilio Ruiz y Salazar ou Mariano Carderera (1815-1893), directeur de l'instruction publique au ministère du Fomento à Madrid.

La place de l'Allemagne mérite un commentaire, d'autant qu'elle reste tout aussi timorée dans les deux premières catégories. Daguet (1886) résume ces timides relations en ces mots: "Nos échanges avec l'Allemagne ne sont malheureusement pas aussi nombreux que nous l'eussions désiré et que nous le faisaient espérer le besoin de savoir et l'universalité attribués aux Allemands. Nous n'eus- 
sions pas cru possible, par exemple, que le rédacteur en chef d'une feuille pédagogique importante de la Bavière, put répondre à une demande d'échange par un refus accompagné de l'explication suivante: "Nous ne savons pas assez le français pour tirer parti de votre feuille"» (p. 4). Il partage également l'avis de Seyffarth, l'éditeur allemand des œuvres de Pestalozzi, lorsque celui-ci s'élève contre un certain M. Lemang de Berlin, «qui propose la Chine pour modèle aux pédagogues allemands et veut élever une muraille impossible autour de la Germanie pour n'y laisser pénétrer aucune idée étrangère» (Daguet, 1880b, p. 366).

Par ailleurs, il est opportun de souligner le "problème Herbart» qui apparaît à plusieurs reprises dans $L ' E ́ d u c a t e u r$, le philosophe allemand étant globalement rejeté par nombre d'instituteurs romands. Daguet (1881) écrit que «l'un de nos anciens et plus expérimentés collaborateurs, M. Xavier Ducotterd, professeur à Francfort, avait promis de nous initier aux idées de Herbart; mais il a reculé devant le peu d'attention que la philosophie pédagogique a pour nos pays français, où elle est volontiers taxée de doctrine germanique et nébuleuse» (p. 67). C'est donc le nouveau rédacteur de L'Éducateur, François Guex, qui mettra le cap sur l'herbartisme dès 1899 , profitant du virage psychologique suscité par Alexander Bain, Herbert Spencer et Herbart lui-même.

Expositions universelles/nationales et congrès pédagogiques

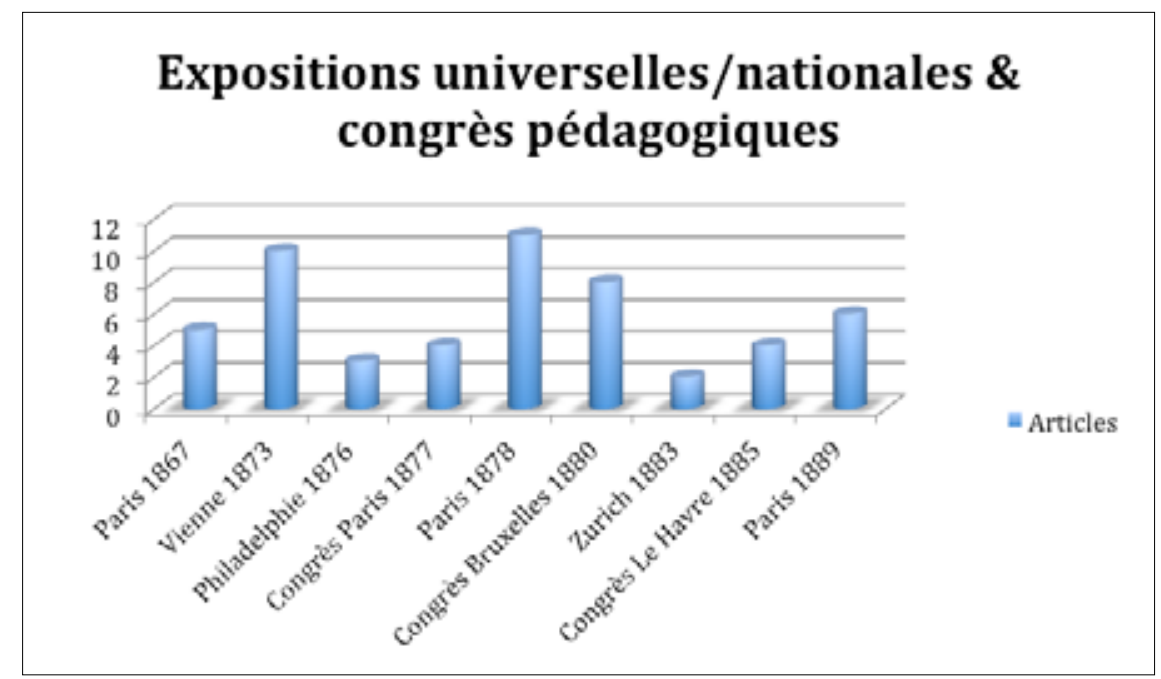

Tableau 4 : Expositions universelles/nationales et rapport de congrès pédagogiques

La dernière catégorie rassemble une part importante des articles dévolus à l'étranger (près de $40 \%$ ). Il faut dire que ce sont clairement les événements internationaux qui fabriquent de l'international dans L'Éducateur. Les exposi- 
tions universelles sont minutieusement étudiées, même si l'on remarque une tendance quelque peu syncrétique à rendre compte de l'exposition suisse au travers de lunettes étrangères ${ }^{10}$. Il s'avère que ce sont toujours les mêmes nations qui nourrissent les commentaires (Prusse, Saxe, Suède, États-Unis, France, Angleterre).

En outre, les expositions internationales jouissent d'une meilleure couverture que les nationales. L'exposition nationale de Zurich (1883) est boudée par L'Éducateur, alors que la Revue pédagogique et le Manuel général de l'instruction primaire lui consacrent plusieurs articles. Enfin, soulignons le travail de Caroline Progler qui devient la correspondante de $L^{\prime}$ Éducateur pour les expositions universelles et les congrès internationaux de l'enseignement depuis 1878 . Ce constat démontre à nouveau que les femmes sont des médiatrices privilégiées de la circulation des savoirs pédagogiques et que l'étude de leurs trajectoires est d'un intérêt déterminant pour la recherche des transferts culturels pédagogiques.

\section{Conclusion}

Au terme de son mandat de rédacteur en chef, Daguet a donc réussi son pari d'ouverture, qui demeure le résultat de vingt ans d'échanges progressifs, de rapprochements et de coopérations entre L'Éducateur et les feuilles pédagogiques occidentales. En 1890, les chroniques helvétiques côtoient toutes sortes d'informations relatives à l'enseignement pratiqué à l'étranger. On remarquera toutefois qu'à l'exception d'articles dévolus aux deux Amériques, les références étrangères qui circulent dans $L^{\prime} E$ ducateur sont essentiellement eurocentrées. En regard de cette palette fort métissée, la revue avait pour les uns perdu son caractère romand et suisse, pour les autres activé une sorte de missionnariat pédagogique:

Votre revue pédagogique, si dignement dirigée, si savamment rédigée au grand avantage des apôtres de la sainte mission de l'enseignement, se peut bien nommer décidément plus internationale que suisse, et en effet elle parcourt, dans son voyage d'irradiation comme la lumière, nombre de pays non seulement en Europe, mais encore au-delà de l'Océan (Zuccaro, 1880).

Daguet (1882) se défend de cette ouverture: «Malgré la part assez grande faite à la Pédagogie étrangère dans nos colonnes, la place la plus considérable, la place d'honneur, on peut dire, n'en appartient pas moins toujours à la pédagogie suisse» (p. 260). Toutefois et en dépit de cette résistance de façade, on ne peut plus douter du fait que la lente construction du système pédagogique romand et plus généralement des systèmes pédagogiques nationaux résulte d'absorptions plus ou moins dissimulées et demeure le fruit d'un phénomène éminemment transnational. Au demeurant, il serait fort intéressant de prolonger les investigations dans d'autres revues helvétiques, afin de préciser les enjeux qui poussent un contexte culturel à emprunter des savoirs configurés dans un autre espace et 
d'analyser leur mode d'acculturation.

En interrogeant les pratiques et savoirs étrangers qui ont circulé dans $L^{\prime} E ́ d u$ cateur, en questionnant la fonction nodale de ses passeurs et médiatrices, on remarque que la structure pédagogique proposée par L'Éducateur s'est constamment nourrie de l'altérité dont une des fonctions principales fut de légitimer les réformes projetées par les cadres romands. Cette perspective d'une «pensée métisse» (Gruzinski, 1999) invite les chercheurs en sciences de l'éducation à suivre la trajectoire des idées présentes dans les revues pour déconstruire et décloisonner les «modèles» nationaux. Plus qu'une recherche des différences, cette démarche cherche à reconstruire les inclusions et les branchements (Amselle, 2005), témoins d'une construction collective de la pédagogie occidentale.

\section{Notes}

1 Cet article est un prolongement du chapitre cinq de la thèse de Fontaine (2013), accessible sur http://archive-ouverte.unige.ch/unige:30434. Nos chaleureux remerciements vont à Béatrice Haenggeli-Jenni et Gregory Durand ainsi qu'aux membres de l'équipe Erhise pour leurs pertinentes relectures.

2 Cette collection, à la formation de laquelle Jean-Jacques Rapet (1805-1882) a consacré cinquante années de sa vie, comprend environ 8000 ouvrages, en plusieurs langues et dont plusieurs sont introuvables. On n'y compte pas moins de 284 ouvrages relatifs à Pestalozzi et à sa méthode, avec des notes du plus haut intérêt. Lors de l'exposition qui fut faite à Zurich, la ville natale de l'illustre pédagogue suisse, pour le centenaire de Pestalozzi, en 1927, on n'avait pu rassembler à grand-peine que 210 ouvrages formant la bibliothèque pestalozzienne. Notons que Rapet fut également l'interprète du Père Girard en France.

3 Sur la circulation et l'acculturation de savoirs pédagogiques dans l'espace franco-romand, voir Ognier (1988), Hofstetter, Magnin, Criblez \& Jenzer (1999), Matasci (2011) et Fontaine (2013).

4 Le premier comité directeur de L'Éducateur rassemble des instituteurs fribourgeois libéraux-nationaux regroupés autour de leur rédacteur Alexandre Daguet. Sur l'histoire de la revue, voir Durand (2013).

5 Sur le premier itinéraire d'Alexandre Daguet, voir Fontaine (2005).

6 Au sujet de la baronne prussienne Bertha von Marenholtz-Bülow (1810-1893), nous savons qu'elle profita de l'appui d'Edgar Quinet et de Jules Barni - deux irréconciliables exilés en Suisse romande - afin d'approcher la tribune du congrès social de Berne de 1865 et y exposer les principes du Kindergarten. Elle y rencontre «le professeur Desor, le savant géologue neuchâtelois, l'historien Daguet et quelques autres, mais elle est déçue, choquée surtout par les diverses professions de foi matérialistes qui lui font qualifier le congrès de "foire des idées" (Du Pasquier, 1959, p. 216).

7 Numa Droz (1844-1899), futur conseiller fédéral, y joua un rôle décisif. Quant à Alexandre Daguet, il n'est, selon nos sources, nullement impliqué dans la SIR jusqu'à ce que l'on fasse appel à lui pour diriger L'Éducateur.

8 Le corps enseignant fribourgeois ne réintégrera la Société des enseignants romands qu'en 1969.

9 Par "contribution", nous entendons une communication écrite d'environ 3 à 5 pages parue dans un numéro de L'Éducateur. Certaines thématiques peuvent se décliner sur plusieurs semaines et donnent lieu à une série d'articles qui sont dès lors comptés séparément dans notre compilation.

10 C'est notamment le cas dans Daguet (1874, 1884, 1885). 


\section{Références}

Amiel, H.-F. (1865). Lettre à Alexandre Daguet. Genève, 17 novembre 1865. Bibliothèque publique de Genève, salle des manuscrits, Archives Amiel 92, Ms fr 3092, 212.

Amselle, J.-L. (2005). Branchements: Anthropologie de l'universalité des cultures. Paris: Flammarion.

Barras, J.-M. (2005). Au temps de l'école normale. D'Hauterive à Fribourg: chronique, contexte et témoignages. Fribourg: Imprimerie St-Paul.

Bugnard, P.-P. (1998/1999). Visiter une cathédrale pour en retrouver le sens. Annales fribourgeoises, tirage à part du tome LXIII, 131-185.

Buisson, F. (1878). Rapport sur l'instruction primaire à l'exposition universelle de Philadelphie. Paris: Imprimerie Nationale.

Caspard, P. (Éd.). (1981). La presse d'éducation et d'enseignement, XVIIIe siècle-1940, tome I. Paris: INRP, Éditions du CNRS.

Choppin, A. (2008). Le manuel scolaire, une fausse évidence historique. Histoire de l'éducation, 117, 7-56.

Compte-rendu du VIIIe congrès scolaire de la société des instituteurs de la Suisse romande à Neuchâtel tenu les 25 et 26 juillet 1882. Neuchâtel: Imprimerie L.-A. Borel.

Compte-rendu du Xe congrès scolaire de la Société des instituteurs de la Suisse romande réuni à Porrentruy les 8, 9 et 10 août 1886. Porrentruy: V. Michel.

Daguet, A. (1865). Congrès des instituteurs suisses à Soleure. L'Éducateur, 4, 58-59.

Daguet, A. (1866). Rapport sur l'origine et la marche de la Société des Instituteurs de la Suisse romande. In Rapport sur la deuxième session de l'assemblée générale des instituteurs de la Suisse romande réunis le 6 août. Fribourg: Imprimerie Ch. Marchand.

Daguet, A. (1867). Les jardins d'enfants. L'Éducateur, 18, 282-285.

Daguet, A. (1869). Union de la Suisse française et de la Suisse allemande. L'Éducateur, 14, 217-219.

Daguet, A. (1871a). Coup d'œil sur l'histoire de la pédagogie depuis les temps anciens jusqu'à nos jours. L'Éducateur, 5, 65-69.

Daguet, A. (1871b). Nécrologie de Joseph-Antoine Henne. L'Éducateur, 6, 85-87.

Daguet, A. (1874). Rapport de M. Sante Polli, de Milan sur l'exposition scolaire suisse à Vienne. L'Éducateur, 24, 377-381.

Daguet, A. (1876-1877). Biographie populaire des pédagogues suisses. Wehrli, l'éducateur thurgovien, le père de l'école des pauvres. L'Éducateur, 23/1876, 353-356; 24/1876, 369-373 et 1/1877, 4-7.

Daguet, A. (1880a). Mission de la presse scolaire: l'importance de cette dernière pour l'instituteur. L'Éducateur, 4, 49-50.

Daguet, A. (1880b). Chronique générale de l'instruction populaire en Europe et dans d'autres continents. L'Éducateur, 23, 365-367.

Daguet, A. (1881). Pédagogie suisse et allemande. L'Éducateur, 5, 67-68.

Daguet, A. (1882). Rapport sur la marche de L'Éducateur 1880, $1881 \& 1882$. L'Éducateur, $17,257-262$.

Daguet, A. (1884). L'exposition scolaire de Zurich, jugée par un homme d'école italien et traduit de l'italien et annoté. L'Éducateur, 1, 3-8.

Daguet, A. (1885). Exposition nationale de Zurich, jugée par M. Dumesnil, Conseiller d'État de la République française. L'Éducateur, 7, 103-107.

Daguet, A. (1886). Pédagogie de l'Allemagne. L'Éducateur, 1, 4-6.

Dubois, P. (1996). La pédagogie catholique dans le "Dictionnaire de Pédagogie" de Ferdinand Buisson. In Pédagogie chrétienne, pédagogues chrétiens, colloque international d'Angers de septembre 1995 (pp. 323-333). Paris: Editions Don Bosco.

Dubois, P. (2000). Le Dictionnaire de F. Buisson et ses auteurs (1878-1887). Histoire de l'éducation, 85, [en ligne] http://histoire-education.revues.org/index1233.html. 
Du Pasquier, M. (1959). Edgar Quinet en Suisse. Douze années d'exil (1858-1870). Neuchâtel: Éditions de la Baconnière.

Durand, G. (2013). L'Éducateur, quels apports pour l'histoire du Syndicat romand? Mémoire de master, Université de Genève (FPSE).

Espagne, M. (1999). Les transferts culturels franco-allemands. Paris: PUF.

Fontaine, A. (2005). Alexandre Daguet (1816-1894): racines et formation d'un historien libéral-national oublié. Mémoire de licence/master, Université de Fribourg (accessible sur http://unige.academia.edu/AlexandreFontaine).

Fontaine, A. (2013). Transferts culturels et déclinaisons de la pédagogie européenne. Le cas francoromand au travers de l'itinéraire d'Alexandre Daguet (1816-1894). Thèse de doctorat, Université de Fribourg et Université de Paris 8 (accessible sur http://archive-ouverte. unige.ch/unige:30434).

Gruzinski, S. (1999). La pensée métisse. Paris: Fayard.

Guillaume, J. (1911). Fröbel. Nouveau Dictionnaire de pédagogie et d'instruction primaire publié sous la direction de F. Buisson. Paris: Hachette.

Hofstetter, R., Magnin, C., Criblez, L. \& Jenzer, C. (Éd.). (1999). Une école pour la démocratie. Naissance et développement de l'école primaire publique en Suisse au 19 siècle. Berne: Lang.

Hofstetter, R. (2010). Genève: creuset des sciences de l'éducation (fin du XIX'e-première moitié du $X X^{e}$ siècle. Genève: Librairie Droz.

Matasci, D. (2011). L'école républicaine et l'étranger. Acteurs et espaces de l'internationalisation de la "réforme scolaire" en France (1870-première moitié du XXe siècle). Thèse de doctorat, Université de Genève.

Nora, P. (Dir.). (1984). Les lieux de mémoire, I. La République. Paris: Gallimard.

Ognier, P. (1988). L'école républicaine française et ses miroirs. Berne: Peter Lang.

Progler, C. (1882). Le centenaire de Frédéric Frœbel. L'Éducateur, 5, 65-68.

Savary, E. (1914). La Société pédagogique de la Suisse romande (1864-1914): notice historique rédigée à l'occasion du jubilé cinquantenaire de la Société. Lausanne: Imprimeries réunies.

Schärer M. E. (2008). Friedrich Frcebel et l'éducation préscolaire en Suisse romande: 1860-1925. Lausanne: Les Cahiers de l'ÉÉSP.

Zuccaro, L. (1880). Lettre à Alexandre Daguet, envoyée de Milan le 12 mars 1880. L'Éducateur, 8, 116-117.

Mots-clés: L'Éducateur, transferts culturels, circulation des savoirs, presse pédagogique, Suisse romande

\section{Eine Zeitschrift «à l'affût du monde»? L'Éducateur als Relais pädagogischer Transfers und Verbindungen in der französischen Schweiz (1865-1890).}

\section{Zusammenfassung}

Der Artikel untersucht verschiedene Arten von Wissenstransfers in der zweiten Hälfte des 19. Jahrhunderts in der französischen Schweiz. Indem L'Éducateur, die Zeitschrift des Lehrerverbands der französischen Schweiz, im Zentrum der Aufmerksamkeit steht, können in diesem entscheidenden pädagogischen Netzwerk regionale sowie transnationale Anleihen dekonstruiert und sowohl Widerstände als auch der Einsatz, der in dieser Wissenszirkulation auf dem Spiel steht, analysiert werden. 
Schlagworte: L'Éducateur, Kulturtransfer, Wissenszirkulation, pädagogische Zeitschriften, Westschweiz.

\section{Una rivista all'ascolto del mondo? L'Éducateur comme veicolo di trasferimento del sapere e di meticciaggi pedagogici in Svizzera Romanda (1865-1890).}

\section{Riassunto}

L'articolo analizza le modalità di trasferimento del sapere avvenute durante la seconda metà del $19^{\circ}$ secolo in Svizzera romanda. Concentrando l'attenzione sulla rivista dei maestri romandi fondata nel 1865 ( $L^{\prime} E$ ducateur), l'articolo analizza i fenomeni dei "prestiti educativi», sia regionali che internazionali. L'importanza della circolazione del sapere e le correlate complesse dinamiche di distribuzione, piu o meno fluide, vengono così messe in evidenza attraverso la descrizione dell'efficace rete di distribuzione delle riviste pedagogiche.

Parole chiave: L'Éducateur, trasferimenti culturali, circolazione dei saperi, stampa pedagogica, Svizzera romanda.

\section{A journal on the lookout for the world? L'Éducateur as relay of transfers and pedagogical mixing in French-speaking Switzerland (1865-1890).}

\section{Summary}

This article aims at analyzing the modes of knowledge transfers that take place during the second part of the 19th century in French-speaking Switzerland. It puts L'Éducateur - journal of the French-speaking teachers founded in 1865 - at the heart of the questioning. It comes to deconstruct the phenomena of borrowing, both at a regional and transnational level, and to show resistances and central stakes of knowledge circulation through the powerful network of pedagogical journals.

Key words: $L ' E ́$ ducateur, cultural transfers, knowledge circulation, pedagogical press, French-speaking Switzerland. 